\title{
Quantum confinement of volume plasmons and interband transitions in germanium nanocrystals
}

\author{
P. D. Nguyen, ${ }^{1, *}$ D. M. Kepaptsoglou, ${ }^{2}$ R. Erni, ${ }^{3}$ Q. M. Ramasse, ${ }^{2}$ and A. Olsen ${ }^{1}$ \\ ${ }^{1}$ Department of Physics, University of Oslo, P.O. Box 1048, Blindern, NO-0316 Oslo, Norway \\ ${ }^{2}$ SuperSTEM Laboratory, STFC Daresbury Campus, WA4 4AD Warrington, Cheshire, United Kingdom \\ ${ }^{3}$ Electron Microscopy Center, Empa, Swiss Federal Laboratories for Materials Science and Technology, CH-8600 Dübendorf, Switzerland
}

(Received 2 October 2012; published 17 December 2012)

\begin{abstract}
The plasmonic properties of individual quantum-sized Ge nanocrystals (NCs) were observed and systematically analyzed by aberration-corrected scanning transmission electron microscopy (STEM) and electron energy loss spectroscopy (EELS). For this purpose, Ge NCs embedded in an $\mathrm{SiO}_{2}$ matrix with controllable size, density, and structure were fabricated using magnetron sputtering. The size dependence of the Ge plasmon energies in the size range of 5-9 $\mathrm{nm}$ is shown to be well depicted by the so-called medium quantum confinement (QC) model, with an effective mass of $0.57 m_{0}$ (contrary to expectations of a stronger quantum effect). In the very low-loss region of the EEL spectra, an apparent blue shift of the $E_{2}$ interband transition peak up to $2 \mathrm{eV}$ and a strong reduction in the oscillator strength were measured for the NCs in the size range of 4-6 nm. It indicates for this smaller size range a transition to a QC regime where the band structure and the density of states are modified dramatically. These trends are explained by a combination of low-loss and core-loss EELS results, which show that the Ge $\mathrm{NCs}$ are surrounded uniformly by nearly stoichiometric $\mathrm{SiO}_{2}$. This local chemistry is shown to provide an infinite potential barrier and to confine electrons and holes in the spherically shaped Ge NCs. In addition to pure QC effects in the Ge NCs, the $\mathrm{SiO}_{2}$ matrix thus plays an important role in the strength of the observed QC and interband transitions.
\end{abstract}

DOI: 10.1103/PhysRevB.86.245316

PACS number(s): 73.22.-f, 73.21.La, 73.63.Bd

\section{INTRODUCTION}

Ge and Si nanocrystals (NCs) embedded in dielectric matrices such as $\mathrm{SiO}_{2}$ have attracted a great deal of attention due to their tailorable electronic and optical properties for a wide range of optoelectronic and solar cell applications. ${ }^{1}$ It is well demonstrated that the electronic structure of the valence band and the conduction band are strongly modified in these NCs. ${ }^{2,3}$ For example, by engineering the sizes and morphologies of $\mathrm{Si}$ and $\mathrm{Ge}$ nanostructures, a widening of effective band gaps and an efficient interband absorption over a certain spectrum range can be obtained. ${ }^{4}$ Therefore, in order to tailor these systems for optoelectronic applications, it is extremely important to understand how their properties evolve as a function of NC size and structure.

If the "particle in a box" model is applied to the aforementioned systems, one would expect the band gap to increase and the characteristic transition energies, such as excitons, to move to higher energies due to the quantum confinement (QC) effects in the confined directions. ${ }^{5}$ The consequences of these effects have been studied extensively by a variety of techniques, including theoretical calculations, ${ }^{3,5,6}$ a combination of lowloss and core-loss STEM-EELS, ${ }^{7-9}$ and $\mathrm{x}$-ray absorption spectroscopy (XAS). ${ }^{10}$ More complicated quantum behaviors of different interband transitions away from the Brillouin zone center of Ge atomic-like discrete structures, which cannot be described completely by the "particle in a box" model, have also been studied through optical absorption, Raman scattering, and ellipsometry. ${ }^{11,12}$ Although these optical methods are commonly used to characterize the optoelectronic properties of such systems, the quantitative information they collectively yield is difficult to interpret due to several factors contributing to the uncertainty of experimental results: size dispersion of $\mathrm{NCs}$, different shapes, density, and interface structures of NCs as well as electron, photon, and phonon transfer between NCs.
Consequently, there have been many apparently conflicting reports on these systems. For instance, Tognini et al. ${ }^{11}$ reported absorption spectra from Ge NCs showing a blue shift of the $E_{2}$ transition, which occurs near the $X$ point of the Brillouin zone, and a weakening of the $E_{1}$ transition, which is along the $\Lambda$ direction, with decreasing Ge NC size. By contrast, Heath et al. ${ }^{12}$ studied extinction spectra from Ge NCs and suggested that $E_{1}$ and $E_{2}$ remain unaffected by QC. Finally, Melnikov and Chelikowsky ${ }^{13}$ calculated absorption spectra of Ge NCs using ab initio calculations and showed that both $E_{1}$ and $E_{2}$ energies are expected to blue shift owing to QC. The discrepancies between the reported experimental results and theoretical calculations demand further investigation in order to predict more accurately the quantum behaviors of these transitions.

One of the most important factors for consideration, when studying QC effects experimentally, is the spread in NC sizes in the samples, which can lead to uncertainties in exciton line positions and widths. ${ }^{10}$ Among the variety of available experimental techniques, aberration corrected STEM-EELS is particularly suited to probing the local electronic structure and elemental distribution inside the individual NCs and their surrounding matrices thanks to its key advantage of very high combined spatial and energy resolutions. ${ }^{14}$ The low-loss part of the EELS spectrum provides inherent information about plasmon densities and band structures of semiconducting and insulating materials. ${ }^{15}$ In addition, the energy-loss near edge structure (ELNES) in core-loss EELS reflects the local density of empty states above the Fermi level, which provides information about the chemistry and bonding of the material under investigation. ${ }^{16}$ In the present paper we combine experimental low-loss and core-loss STEM-EELS to study a number of phenomena related to $\mathrm{QC}$ in individual Ge NCs embedded in an $\mathrm{SiO}_{2}$ matrix. The observed phenomena include an apparent blue shift in plasmon energies and an enhancement 
of $\mathrm{Ge}$ interband transitions with decreasing $\mathrm{NC}$ size. In order to facilitate the interpretation of the experimental results, theoretical simulations have also been carried out for similar confined nanostructures to predict possible behaviors of the excitations in the low-loss EELS and thus provide a clearer physical understanding. In addition to the quantum size effects, chemical states and electronic properties of the $\mathrm{SiO}_{2}$ matrix as well as effects due to changes in the Ge crystal structure are also investigated and discussed.

\section{EXPERIMENT}

A four-layer structure thin film composed of an $\mathrm{SiO}_{2}$ buffer layer followed by two $\mathrm{SiGeO}$ middle layers with different Ge contents and a capping $\mathrm{SiO}_{2}$ layer was deposited on a (100)-oriented single-crystal Si substrate by radio frequency (rf) magnetron sputtering in Ar atmosphere. These layers will be hereafter named as layers 1, 2, 3, and 4 for simplicity. Prior to sputtering, the chamber was evacuated to $5 \times 10^{-7}$ mbar. During the film deposition the Ar pressure in the chamber was kept at $1 \times 10^{-3}$ mbar. Layers 2 and 3 of $\mathrm{SiGeO}$ were obtained by co-sputtering and deposition from $\mathrm{SiO}_{2}$ and $\mathrm{Ge}$ targets without any intentional substrate heating. The different Ge content between these layers was achieved by adjusting the sputtering rate for the Ge target, while the sputtering rate for the $\mathrm{SiO}_{2}$ target was kept constant. With the purpose of forming $\mathrm{Ge} \mathrm{NCs}$ in the oxide matrix, the sample was subsequently annealed at $800{ }^{\circ} \mathrm{C}$ for $1 \mathrm{~h}$ in a sealed $\mathrm{SiO}_{2}$ glass tube evacuated to a vacuum of better than $1 \times 10^{-2}$ mbar. High-resolution transmission electron microscopy (HRTEM) observations were performed at $200 \mathrm{keV}$ primary beam energy with a JEOL $2010 \mathrm{~F}$ microscope. The STEM-EELS experiments were performed at $60 \mathrm{keV}$ in a $C_{s}$-corrected Nion UltraSTEM $^{17}$ equipped with a Gatan Enfina spectrometer. The cold field electron emitter of this instrument has a native energy spread of $0.35 \mathrm{eV}$, as determined from the full width at half maximum of the zero-loss peak in typical operating conditions. The EELS experiments were carried out with a probe size of $1.1 \AA$, the collection semiangle was $4 \mathrm{mrad}$ for the low-loss data sets and $32 \mathrm{mrad}$ for the core-loss data sets. The probe convergence semiangle was $30 \mathrm{mrad}$. Both 2D-EELS spectrum imaging and distributed-dose "SMART EELS"18 line-scan techniques were employed. The latter was used in order to mitigate the effects of radiation damage and contamination by distributing the electron dose received by individual NCs. Postmortem images were systematically acquired in order to check for possible specimen drift during the data collection and to assess specimen beam damage.

\section{RESULTS AND DISCUSSION}

\section{A. Structural and chemical characterization}

HRTEM imaging and electron diffraction confirmed the formation of Ge NCs in both layers 2 and 3. As shown in Fig. 1, layer 2 has a higher density and bigger grain size of Ge NCs $(5-10 \mathrm{~nm})$ than layer $3(2-5 \mathrm{~nm})$. For both layers, the NCs are nearly spherical in shape and uniformly distributed in the bulk of these layers. The results of quantitative energy dispersive $\mathrm{x}$-ray spectroscopy analysis showed that the concentration of elemental Ge in layers 2 and 3 are $40 \%$ and $12 \%$, respectively.

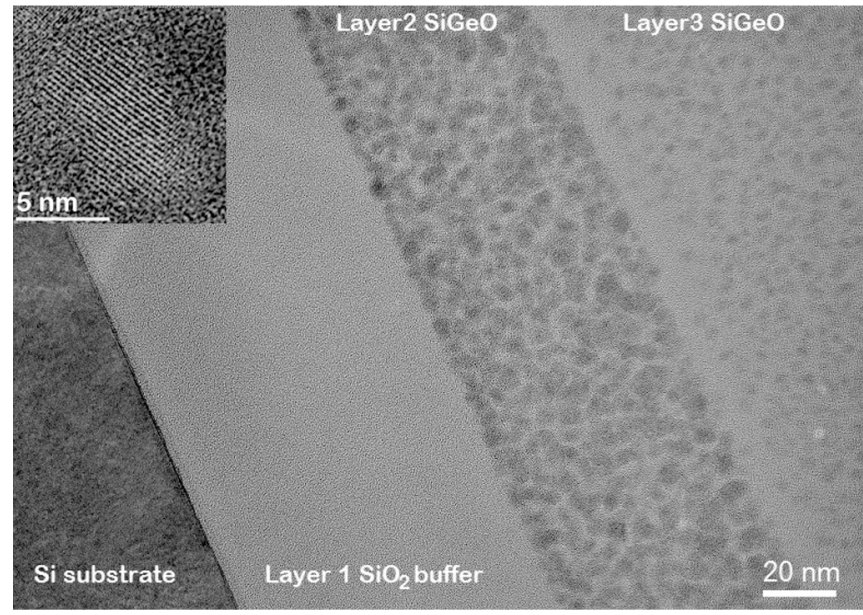

FIG. 1. HRTEM image of the three layers: layer 2 has a higher density and bigger grain size of Ge NCs $(5-10 \mathrm{~nm})$ than layer 3 $(3-5 \mathrm{~nm})$. The inset shows clear lattice fringes of a Ge NC in layer 2.

Some of the NCs, which precipitated densely in layer 2, are interconnected and/or formed twinning defects, while all of the NCs in layer 3 are well separated and defect-free. In addition, for layer 3 the lattice fringes inside the NCs and the interfaces between them and the matrix were not resolved in the HRTEM images as clearly as those in layer 2. Especially the particles with sizes $\sim 2 \mathrm{~nm}$ in layer 3 were found to be amorphous, which is in good agreement with XAS results reported by Araujo et al. ${ }^{19}$ on Ge NCs implanted in an $\mathrm{SiO}_{2}$ matrix, and molecular-dynamics results for Ge NCs embedded in an amorphous matrix reported by Bording and Taft $\varnothing .^{20}$

It is well known that the optical properties of $\mathrm{Si}$ and $\mathrm{Ge} \mathrm{NCs}$ are strongly affected by the structural and chemical properties of their surfaces since NCs have a high surface to volume ratio. ${ }^{21}$ The interface between a NC and its surrounding matrix can play a crucial role in the optoelectronic properties of the NCs. ${ }^{22,23}$ By analyzing the $\mathrm{Si} L_{2,3}$ edge from the core-loss EELS line scans across the surrounding matrix to the NC core, useful information about the local bonding, defect, and chemical state at the site where the atom is being excited can be obtained. Figure 2(a) shows the HAADF STEM survey image of a Ge particle in layer 2 where the trace of a core-loss EELS line scan taken across the particle is marked from A to D. Figure 2(b) shows background-subtracted, averaged EELS spectra of the Si $L_{2,3}$ edge (each spectrum in this figure is an average of 20 adjacent spectra) from the four probe positions indicated in Fig. 2(a). The spectra correspond to the areas marked A to D in Fig. 2(a). Compared to the Si $L_{2,3}$ edge of stoichiometric $\mathrm{SiO}_{2}$ reported by Erni et al., ${ }^{16}$ the four spectra show similar peak energies and ELNES. It is expected that the suboxides $\left(\mathrm{Si}^{1+}, \mathrm{Si}^{2+}\right.$, and $\left.\mathrm{Si}^{3+}\right)$ should show a broad intensity band in front of the $\mathrm{Si} L_{2,3}$ edge. ${ }^{16,24-26}$ As seen in Fig. 2(b), no extra intensity in the pre-edge region is observed. The only difference is found in the intensities of the edges, which are as expected higher in the matrix than in the NC core. Therefore the Ge NCs appear to be surrounded uniformly by nearly stoichiometric $\mathrm{SiO}_{2}$, and if there is any chemical modification in the matrix [e.g., $\mathrm{O}$ deficiency, coexistence of $\left.\left(\mathrm{SiO}_{2}\right)_{x}\left(\mathrm{GeO}_{2}\right)_{\mathrm{y}} \ldots\right]$, the concentration of any secondary phases must be too low to be detected at the $\mathrm{Si} L_{2,3}$ edges. 

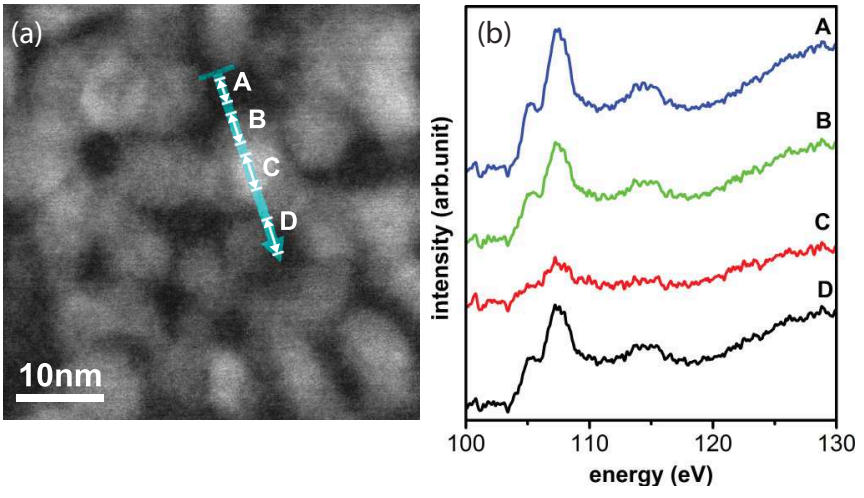

FIG. 2. (Color online) (a) HAADF STEM survey image of a Ge particle in layer 2 where the trace of core-loss EELS line scans taken across the particle is marked from A to D. (b) Backgroundsubtracted, averaged EELS spectra of the Si $L_{2,3}$ edge from the four probe positions indicated in (a). The spectra correspond to the areas marked A to D in (a), respectively.

A similar behavior was observed for the averaged core-loss EELS line scans across the film structure from layer 1 to 3 , at both $\mathrm{Si} L_{2,3}$ and $\mathrm{O} K$ edges. The intensities of these edges are highest in layer 1 of pure $\mathrm{SiO}_{2}$ and lowest in layer 2 where Ge NCs precipitated densely. The fact that the similar shape and sharp features of the Si $L_{2,3}$ edge can be observed in the three layers indicates that the suboxide content is negligible and the chemical structure of the matrix, which is stoichiometric $\mathrm{SiO}_{2}$, is independent of the Ge content in layers 2 and 3. This information is essential for deconvoluting the contribution of the matrix in the low-loss EELS data that will be discussed in the following section of this paper.

\section{B. Experimental and calculated low-loss EELS}

The controllable sizes, spatial distributions, and structures of Ge NCs in layers 2 and 3 allow us to study the size-dependent volume plasmon and interband transition energies of individual NCs in a large range of sizes with different crystal densities and structures under the same experimental conditions. Due to the radiation damage sensitivity of the ${\mathrm{Ge}-\mathrm{SiO}_{2}}_{2}$ system and in order to improve the signal to noise ratio, the distributeddose SMART EELS acquisition technique was employed for low-loss EELS. The typical setting of SMART acquisitions is shown in Fig. 3(a), where the HAADF image is marked with the trace of the line scan taken across a Ge NC in layer 2, while the dose is distributed by displacing the probe along the other direction at each point in the line scan, as indicated. Figure 3(b) shows zero-loss peak subtracted (using a power-law function), averaged plasmon EELS spectra from the line scan in Fig. 3(a). Each spectrum in Fig. 3(b) is an average of 20 adjacent spectra (of a line scan containing a total of 150 spectra). The spectra correspond to the areas marked A to C in Fig. 3(a), respectively. Moving from the matrix to the $\mathrm{NC}$ core, the plasmon peak shifts to lower energy. The plasmon response of this heterostructure is expected to contain contributions from both the Ge NCs and the surrounding $\mathrm{SiO}_{2}$ matrix. Therefore, a deconvolution of these broad plasmon peaks is needed in order to identify the individual constituents. ${ }^{9}$ By using the Voigt functional-form fitting method, different component peaks are resolved for spectrum B taken at the NC core as shown in Fig. 3(c). The two main components under the plasmon peak were assigned to the volume plasmons of the Ge NC and $\mathrm{SiO}_{2}$, matrix respectively. The position of the $\mathrm{SiO}_{2}$ matrix plasmon was verified independently by acquisition of low-loss EEL spectra from the matrix immediately surrounding each studied NC. The plasmon energy of the matrix was found to be in the range of $23-24 \mathrm{eV}$. This is in agreement with the core-loss EELS results, which showed that the matrix composition is uniform and is nearly stoichiometric $\mathrm{SiO}_{2}$. The remaining components correspond to the broad $M_{4,5}$ ionization edge of Ge NCs at around $29 \mathrm{eV}^{27}$ and a broad peak with maximum around $6 \mathrm{eV}$. This peak, seen only in spectra acquired in layer 2 (Fig. 3) and layer 3 (not shown), could have several origins, including interband transitions from Ge NCs, retardation effects, such as Čerenkov radiation and guided light modes, or interface plasmon excitation between Ge NCs and the $\mathrm{SiO}_{2}$ matrix.

In order to determine the origin of the low-loss peak at $6 \mathrm{eV}$ we performed low-loss EELS calculations for a simplified sandwich structure of $\mathrm{SiO}_{2} / \mathrm{Ge} / \mathrm{SiO}_{2}$ as well as for amorphous $\mathrm{SiO}_{2}$ and $\mathrm{Ge}$ of varying thickness, for conditions that reflect
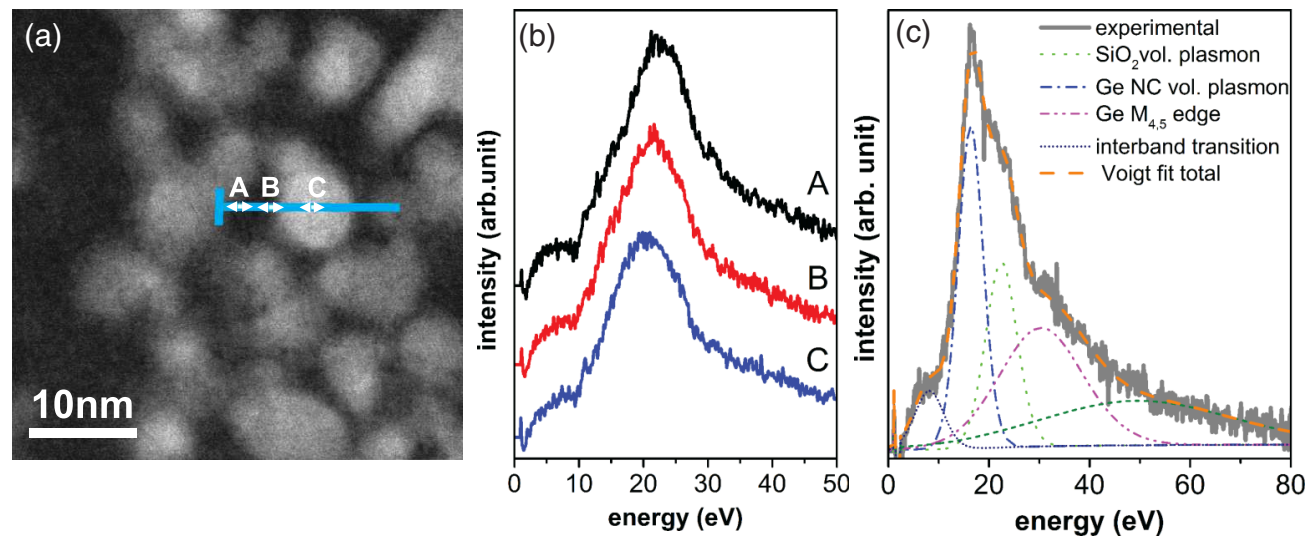

FIG. 3. (Color online) (a) Typical setting of EELS SMART acquisitions for the low-loss line scan, where the HAADF image is marked with the trace of the line scan taken across the Ge NC. (b) Zero-loss peak subtracted, averaged plasmon EELS spectra from the line scan in (a). The spectra correspond to the areas marked A to $\mathrm{C}$ in (a), respectively. (c) Different component peaks are resolved for spectrum $\mathrm{C}$ taken at the $\mathrm{NC}$ core by using the Voigt functional-form fitting method. 


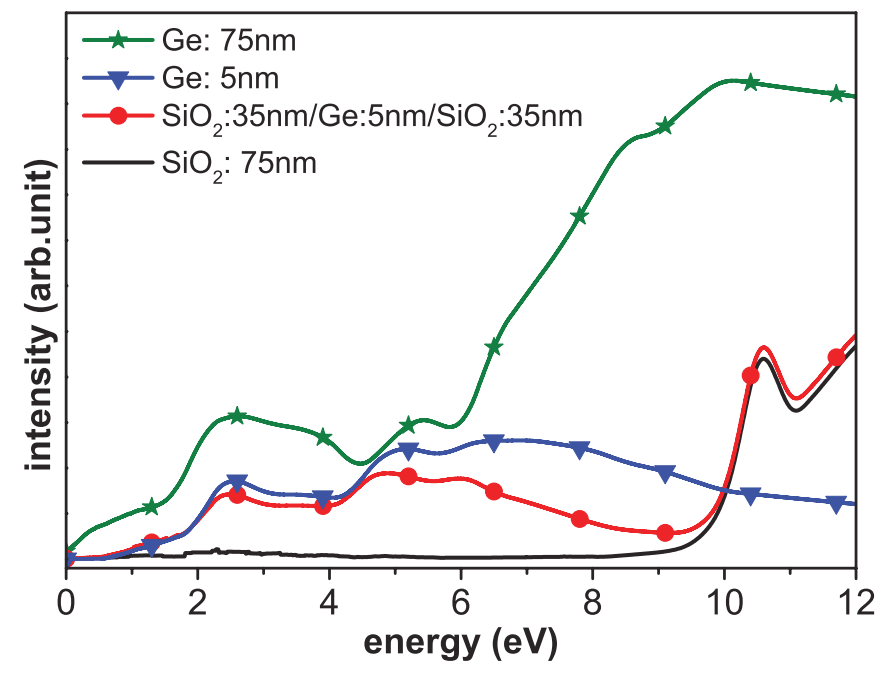

FIG. 4. (Color online) Calculated low-loss EELS spectra at $60 \mathrm{kV}$ for four systems: $75 \mathrm{~nm}$ of $\mathrm{SiO}_{2}, 75 \mathrm{~nm}$ of Ge, $5 \mathrm{~nm}$ of Ge, and $(35 \mathrm{~nm}$ $\mathrm{SiO}_{2} / 5 \mathrm{~nm} \mathrm{Ge} / 35 \mathrm{~nm} \mathrm{SiO} 2$ ). For the last three systems, the interband transition $\sim 2.5$ and $5 \mathrm{eV}$ is well described.

the experimental setup. In these simulations, we use the Ge and $\mathrm{SiO}_{2}$ bulk dielectric functions, ${ }^{28}$ and apply the dispersion bracket formalism of Bolton and Chen ${ }^{29,30}$ which allows for calculating surface/interface and bulk plasmons as well as (relativistic) radiation losses. QC effects are not included as they would require corresponding dielectric data. ${ }^{31,32}$ The calculations show that the very low-loss regions $(0-10 \mathrm{eV})$ of the EELS spectra from the $\mathrm{Ge}$ and $\mathrm{SiO}_{2} / \mathrm{Ge} / \mathrm{SiO}_{2}$ systems are dominated by interband transitions at $\sim 2.5$ and $4.8 \mathrm{eV}$ originating from the thin Ge layers (Fig. 4). These features, which are present in the calculated spectra of a pure Ge slab of thickness 75 and $5 \mathrm{~nm}$, respectively (green and dark blue curves), as well as in the spectrum of the sandwich structure of a $5 \mathrm{~nm}$ thick Ge slab embedded in $70 \mathrm{~nm}$ amorphous $\mathrm{SiO}_{2}$ (red curve), closely resemble the $E_{1}(2.3 \mathrm{eV})$ and $E_{2}$ $(4.4 \mathrm{eV})$ transitions reported in bulk $\mathrm{Ge} .^{33,34}$ No interface plasmons between $\mathrm{SiO}_{2}$ and $\mathrm{Ge}$ thin layers were predicted by the simulation, implying that there is no strong coupling of the dielectric functions. The fact that the features and intensities of the peak around $6 \mathrm{eV}$ observed experimentally in our spectra do not vary as the beam moves from the particle core to the matrix/particle interface further confirms that these features are not likely related to the interface plasmons. Hence, the experimental data can be interpreted in terms of a superposition of a spectrum that is due to the $\mathrm{SiO}_{2}$ matrix and another part that is due to the Ge NCs. Moreover, there are no significant peaks present in the calculated spectra that are due to retardation effects such as Čerenkov losses or losses due to the excitation of guided light modes. Indeed, the absence of significant radiation losses can be expected at a primary electron energy of $60 \mathrm{keV}$. Yet, what cannot be completely ruled out is that residual radiation damage leads to the creation of electronically active defects in $\mathrm{SiO}_{2}$ that could contribute to the absorption in the sub-10 eV energy range. ${ }^{35-37}$ However, as the spectrum recorded from amorphous $\mathrm{SiO}_{2}$ does not show significant intensity in the sub- $10 \mathrm{eV}$ range, the overall effect of residual radiation damage must be small. Therefore, the experimental spectra reflect the actual dielectric characteristics of the material (the interband transitions from Ge NCs) rather than the interaction with the matrix or electron beam. It is therefore concluded that the peak observed at $6 \mathrm{eV}$ peak more likely corresponds to the $E_{2}$ interband transition, blue shifted with respect to its bulk value.

\section{Quantum confinement of $\boldsymbol{E}_{\mathbf{2}}$ interband transition}

The $E_{1}$ transitions $(\sim 2.3 \mathrm{eV})$ were not observed in our experimental low-loss EELS spectra, possibly due to the weakening of these transitions with the $\mathrm{NC}$ size reduction due to a transfer of oscillator strength from $E_{1}$ to $E_{2}$ transition $^{11}$ and the potential overlap with the tail of the zero-loss peaks. Furthermore, Guerra et al. ${ }^{38}$ reported density functional theory (DFT) calculations on the absorption spectra of $\mathrm{Si} \mathrm{NCs}$ embedded in $\mathrm{SiO}_{2}$ matrix and suggested that the absorption at low energy $(<4 \mathrm{eV})$ is strongly damped due to the screening induced by polarization effects at the NC interfaces. Therefore, it can be expected that the $E_{1}$ transition features tend to vanish in the presence of local field effects at the NC interfaces.

As discussed above, the $6 \mathrm{eV}$ peak can be assigned to the $E_{2}$ interband transition, which is one of the most dominant absorption peaks in the visible region reported in the literature. ${ }^{11,33,34,39}$ Figure 5(b) shows the evolution of the $E_{2}$ peak position with $\mathrm{NC}$ size, as determined by the zero crossing of the first derivative of the low-loss spectrum ${ }^{11,33,39}$ [Fig. 5(a)]. The $E_{2}$ peaks show a very strong size dependence in the size range of $\sim 4-6 \mathrm{~nm}$. Radiation damage in the very small NCs does not permit a more detailed study of the behavior of this transition in the size range lower than $\sim 4 \mathrm{~nm}$. When the NC size decreases from 6 to $4 \mathrm{~nm}$, a dramatic blue shift of the $E_{2}$ peak by $\sim 2 \mathrm{eV}$ is observed, in addition to a depression of its features. A similar behavior of this transition in optical extinction spectra was reported by Hayashi et al. ${ }^{34}$ for sputtered Ge NCs embedded in $\mathrm{SiO}_{2}$ thin films, who suggested that for small Ge NCs $(\sim 2.7$ to $4.2 \mathrm{~nm})$ the whole band structure and the density of states are altered dramatically.
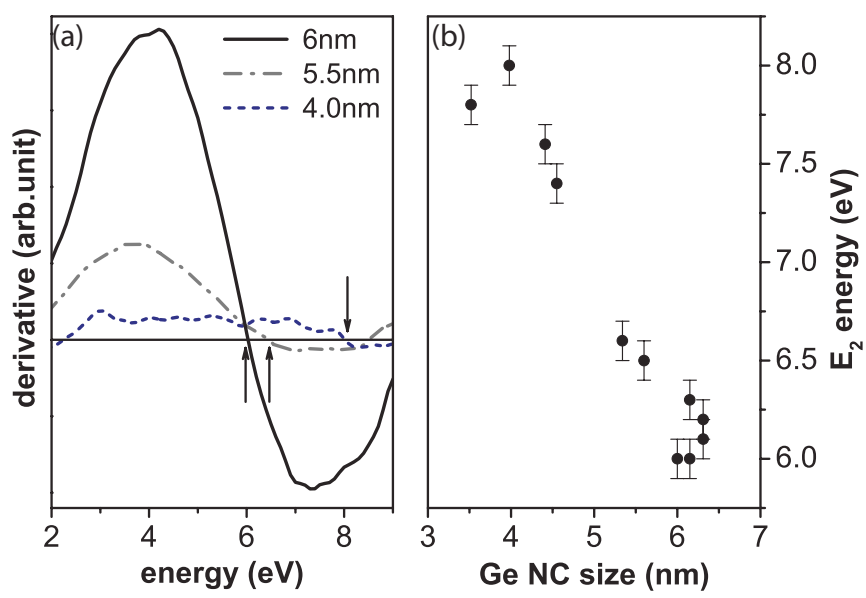

FIG. 5. (Color online) (a) First derivative of $E_{2}$ transition peaks of Ge NCs with different sizes. The zero crossing of the curves determines the shift of $E_{2}$ peaks. (b) The blue shift of $E_{2}$ transitions with decreased $\mathrm{NC}$ size. 
Compared to the shift of the $E_{2}$ transition obtained by $a b$ initio calculations of the absorption spectra for hydrogenated Ge NCs reported by Melnikov et al. ${ }^{13}$ our data show a higher shift. This might be attributable to the propensity of local density approximation calculations to underestimate the energy of the $E_{2}$ transition. ${ }^{13}$ A smaller shift is also found for the optical absorption data measured from Ge NCs confined in $\mathrm{Al}_{2} \mathrm{O}_{3} / \mathrm{Ge}$ multilayer structures by Bottani et al.,${ }^{39}$ or from Ge NCs embedded in $\mathrm{SiO}_{2}$ by Hayashi et al. ${ }^{34}$ albeit with the same trend of size dependence. This can be expected since optical absorption data are acquired with a broader beam over an average of NC size distributions, while our EELS data are obtained directly from individual NCs by using a very small probe size $(\sim 1.1 \AA)$. The impact of using different dielectric matrices and growth methods on the observed quantum size effects is also very important in the interactions between $\mathrm{NCs}$ and matrices, interface structures, induced stress, and local field effects. ${ }^{6,38,40,41}$ As shown by DFT calculations on the absorption spectra of $\mathrm{Si}$ NCs embedded in $\mathrm{SiO}_{2}$ matrix by Guerra et $a l .{ }^{38}$ local field effects cause a blue shift of the main absorption peak $(\sim 5.5 \mathrm{eV})$, in particular for small $\mathrm{NCs}$, which show a sensitivity to their surrounding environment related to perturbations of the absorption resonance conditions. Therefore, we suggest that the higher blue shift of the $E_{2}$ transition observed in our experiments as compared to other reported data ${ }^{13,39}$ can be attributed to the contribution of local field effects at the interfaces between Ge NCs and the $\mathrm{SiO}_{2}$ matrix. Crucially, this enhancement of interband absorption with the reduction of Ge NC sizes and the variations of this absorption with the crystal quality suggest a practical way of tuning the efficiency of interband absorptions over certain spectral ranges.

\section{Quantum confinement of volume plasmon energy}

Depending on the Bohr radius $\left(a_{B}\right)$ and the NC diameter $(d)$, three regimes of QC can be defined by the effectivemass approximation (EMA): 5,6 weak, medium, and strong confinement (for more details, refer to Refs. 5 and 6). For $\mathrm{Ge}, a_{B}$ is $\sim 24 \mathrm{~nm}$, which means that for the size range of Ge NCs in this work $(\sim 2-10 \mathrm{~nm})$ the strong confinement regime would be expected. Moreover, the blue shift of the plasmon energies $E_{P}$ with decreasing particle diameter is attributed to the quantum size effect of the band gap that affects the plasmon energy through the oscillator strength as described by Mitome et al. ${ }^{42}$ Experimental EELS data of $\mathrm{Si} \mathrm{NCs}$ and $\mathrm{Ge}$ nanowires have revealed trends of $E_{P}$ $\propto 1 / d^{2}$ and $E_{P} \propto 1 / d^{1.2}$, respectively. ${ }^{8,9,42}$ Under the same experimental conditions, Si and Ge NCs with similar structure and geometry embedded in a dielectric matrix can be expected to follow the same trend of $E_{P} \propto 1 / d^{2}$. The size dependence of plasmon energies for Ge NCs (Fig. 6) is plotted against theoretically predicted trends in three different regimes: strong, medium, and weak confinement. The theoretical QC curves follow the $E_{P} \propto 1 / \mu . d^{2}$ relation, where $\mu$ is the effective mass of the electron-hole pair in each of these three QC regimes. ${ }^{5,42}$ Overlaying our experimental data points with the theoretical curves, it appears that the strong regime predicted for the NC sizes considered here overestimates the QC effects observed experimentally. The experimental data show instead

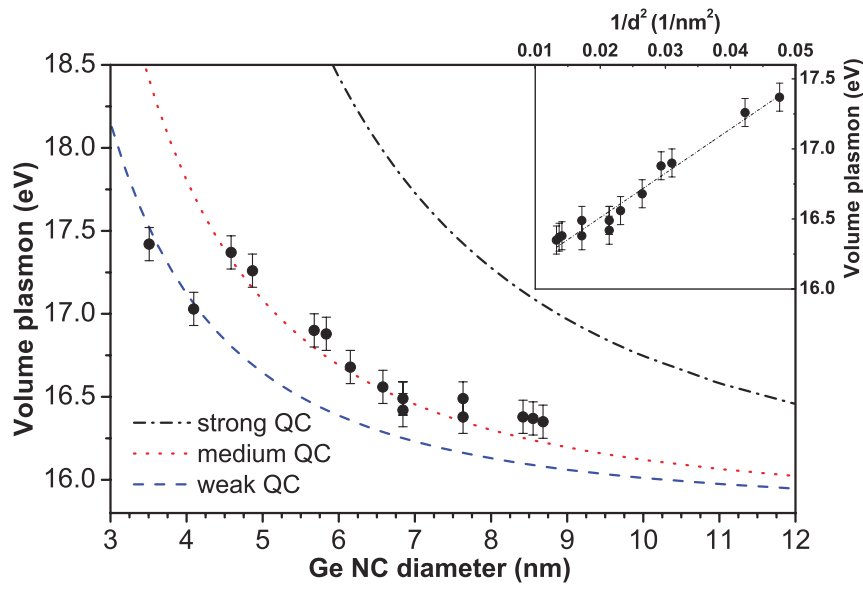

FIG. 6. (Color online) NC size dependence of the Ge volume plasmon energy. The dotted-dashed, dotted, and dashed curves show the theoretically predicted $E_{P} \propto 1 / d^{2}$ relationship in three different regimes: strong, medium, and weak, respectively. The inset shows the data fitting for $\mathrm{NCs}>4 \mathrm{~nm}$.

that most of the NCs in layer $2(>4 \mathrm{~nm})$ follow closely the medium confinement curve and are best fitted by the equation $E_{P}=31.58 / d^{2}+15.87$ (see the inset in Fig. 6), which gives an estimated effective mass $\mu=0.57 m_{0}$, where $m_{0}$ is the electron rest mass, consistent indeed with a medium confinement model. By contrast, the NCs in layer $3(<4 \mathrm{~nm})$ appear to follow the weak confinement curve, although fewer data points are available. The error bars indicate the accuracy in determining the plasmon shift, which was verified statistically from experiments and the instrument resolution.

While neither case appears to follow the initial theoretical expectation of strong confinement behavior, the observed medium confinement trend for the Ge NCs $>4 \mathrm{~nm}$ is in fact in good agreement with the experimental EELS results reported by Mitome et al..$^{42}$ for freestanding Si NCs. They also agree with the effective mass theoretical models calculated by Barbagiovanni et al. ${ }^{6}$ for $\mathrm{Si}$ and $\mathrm{Ge} \mathrm{NCs}$ embedded in an $\mathrm{SiO}_{2}$ matrix. The latter were further confirmed by photoluminescence ${ }^{43}$ and $\mathrm{XAS}^{10}$ data.

It is of interest to compare the strength of $\mathrm{QC}$ effects in the valence band of Ge NCs and of a similar system consisting of Si NCs, as observed through the blue shift of plasmon energies. We recently reported a strong QC in Si NCs embedded in a silicon nitride matrix, ${ }^{9}$ far stronger than the present $\mathrm{Ge} \mathrm{NCs}$ embedded in an $\mathrm{SiO}_{2}$ matrix. While this may appear to contradict earlier theoretical calculations ${ }^{44}$ and experimental XAS results ${ }^{10}$ (which showed stronger QC effects in $\mathrm{Ge}$ than in $\mathrm{Si}$ ), we believe it illustrates the pivotal role of the matrix used when fabricating these devices. ${ }^{45}$ As suggested by Barbagiovanni et al., ${ }^{6}$ the $\mathrm{SiN}_{x}$ matrix acts as a finite potential barrier, allowing for tunneling of carriers; hence, quantum dots in $\mathrm{SiN}_{x}$ can be described quite accurately by a strong confinement model. By contrast, $\mathrm{SiO}_{2}$ acts as an infinite potential barrier ${ }^{6}$ and the confinement strength in this system can be expected to be lower. More importantly, recent DFT calculations have shown that in addition to QC effects in small NCs, the matrix must be taken into account when evaluating the resultant energy levels. ${ }^{46}$ With 
increasing polarity of the bonds between the NCs and the matrix, there is an increasing dominance of the interface strain over the QC effects. Therefore, we suggest that in the system of $\mathrm{NCs}$ embedded in a polar $\mathrm{SiO}_{2}$ matrix, the QC can be drastically reduced as it competes against interface effects.

A combination of factors is likely to contribute to the observed downward shift in volume plasmon energies for the NCs $<4 \mathrm{~nm}$, from medium to weak confinement. First, it has been reported both experimentally and theoretically that interface states could play an important role in NCs smaller than a critical size $(\sim 3 \mathrm{~nm})$, whereas the larger crystals simply follow the usual QC model. ${ }^{46-48}$ Second, there are reports of lattice instability, which could cause a structural transition from a diamond-type structure to an amorphous phase in very small crystallites $(<3 \mathrm{~nm}) .{ }^{49}$ As mentioned earlier, the particles with sizes $\sim 2 \mathrm{~nm}$ observed in layer 3 were found to be amorphous. Bording and Taftø have used moleculardynamics simulations to predict a critical size of $2 \mathrm{~nm}$ for Ge NCs, below which the entire crystal will to some degree be deformed or eventually be assimilated in the amorphous phase. ${ }^{20}$ This transition would very likely change the physical properties related to the electronic band structure. Calculation of Ge band structure modifications caused by phase transitions (using plasmon energy shifts as an indicator) could further confirm this hypothesis. Finally, the higher density and smaller distance between the NCs $>4 \mathrm{~nm}$ in layer 2 could cause stronger coupling effects than in the more sparsely populated layer 3 . This could in turn enhance the optical absorption cross sections and the stronger plasmon coupling between NCs could give rise to the stronger confinement trend in layer 2. This suggestion still remains explorative: further experimental and theoretical studies would be required to reach a more certain conclusion.

\section{E. Comment on the band gap of the $\mathrm{SiO}_{2}$ matrix}

Besides pure NC size effects, the chemistry of the matrix can play an important role in the observed quantum size effects in term of potential barrier height. In order to ascertain the presence of any change in the $\mathrm{SiO}_{2}$ matrix band gap (compared to pure $\mathrm{SiO}_{2}$ ) we compared low-loss EEL spectra from layers 1 and 2 [Fig. 7(a)]. Figure 7(b) shows the corresponding zero-loss peak subtracted plasmon peaks, extracted from the SMART line scan at positions A and B [Fig. 7(a)] and averaged over 20 adjacent data points to improve the signal-to-noise ratio. Spectrum A from layer 1 shows a sharp peak at $24 \mathrm{eV}$ corresponding to the plasmon energy of pure $\mathrm{SiO}_{2}$, while spectrum B from layer 2 shows an asymmetric plasmon peak which is skewed toward the lower energy side, implying a contribution from both $\mathrm{Ge}$ and $\mathrm{SiO}_{2}$ signals. The deconvolution of spectrum $\mathrm{B}$ revealed component peaks at 23 and $17 \mathrm{eV}$ corresponding to the plasmon energies of $\mathrm{SiO}_{2}$ and $\mathrm{Ge} \mathrm{NCs}$, respectively. In addition to the distinct plasmon peaks, the very low-loss energy regions from 4 to $11 \mathrm{eV}$ in spectra $\mathrm{A}$ and $\mathrm{B}$ exhibit significant differences in features and intensities. The visible band gap signal of the stoichiometric $\mathrm{SiO}_{2}$ in spectrum $\mathrm{A}$ is estimated to be at $9.5 \mathrm{eV}$ (from the intersection of a straight line originating from the background level with a linear fit to the onset of the low-loss signal spectrum), while
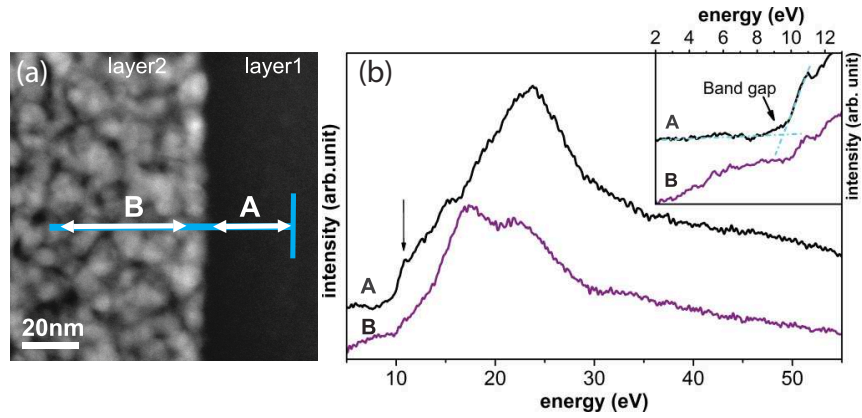

FIG. 7. (Color online) (a) HAADF-STEM image of layers 1 and 2 with different probe positions labeled from which corresponding averaged low-loss EELS SMART line scans were acquired. (b) Plasmon peaks extracted from the SMART line scan at different positions indicated in (a). The inset shows visible band gap signal of the stoichiometric $\mathrm{SiO}_{2}$ in spectrum A which is estimated to be at $9.5 \mathrm{eV}$, while it becomes hardly to recognized in spectrum $\mathrm{B}$. The arrow points out position of the exciton peak observed in both spectra $\mathrm{A}$ and $\mathrm{B}$.

it is no longer visible in spectrum B. This can be attributed to the overlap with the $E_{2}$ interband transition at $\sim 6 \mathrm{eV}$ in front of the band gap onset in spectrum $\mathrm{B}$. Therefore, it is impossible to conclude directly if there is a real change in the $\mathrm{SiO}_{2}$ band gap energy from layer 1 to layers 2 and 3 or whether this is caused by the contribution of Ge NCs to the low-loss spectra. On the other hand, any change of the characteristic exciton peak around $11 \mathrm{eV}^{16}$ just at the band gap signal observed in all of our low-loss EELS spectra might be used as an indirect evidence of a band gap change of $\mathrm{SiO}_{2}$. The exciton peaks measured in layers 1, 2, and 3 are 11.0, 10.8 , and $10.9 \pm 0.3 \mathrm{eV}$ respectively. These values compare well with literature data for crystalline $\mathrm{SiO}_{2}$, which indicate an exciton peak at $10.8 \mathrm{eV} .^{50}$ Thus, if there is a band gap variation of the $\mathrm{SiO}_{2}$ matrix, it is too small to be detected within our experimental error. This is consistent with the core-loss EELS results, which show that the features and peak energies of the Si $L_{2,3}$ absorption edges of layers 2 and 3 do not change compared to that of the stoichiometric $\mathrm{SiO}_{2}$ in layer 1 .

\section{CONCLUSION}

Our studies have shown clear evidence of QC effects on the volume plasmons and interband transitions of individual $\mathrm{Ge}$ $\mathrm{NCs}$ embedded in an $\mathrm{SiO}_{2}$ matrix. We have explained some of the discrepancies between earlier theoretical and experimental works on the behavior of the $E_{2}$ transition, by demonstrating a blue shift of $\sim 2 \mathrm{eV}$ when the NC size decreases from 6 to $4 \mathrm{~nm}$. The theoretical medium confinement curve reproduces well the overall size dependence of plasmon energies, although it appears to overestimate the QC for NCs smaller than $4 \mathrm{~nm}$, which are better described by a weak confinement model. This double regime, as well as the overall weaker confinement effects (a stronger confinement was expected from initial theoretical considerations) can be interpreted as the result of structure reconstruction and enhanced interface effects in the very small NCs. The influence of the $\mathrm{SiO}_{2}$ matrix on the optical response of the NCs was also investigated. The nearly stoichiometric $\mathrm{SiO}_{2}$ matrix is found to provide not only an 
infinite potential barrier to confine electrons and holes in the spherical shape Ge NCs but also an increasing polarity of the bonds at the interfaces between the NCs and the matrix compared to the $\mathrm{SiN}_{x}$ matrix. These factors are very important to determine the strength of the observed QC and the enhanced interband transitions.

\section{ACKNOWLEDGMENTS}

The authors would like to thank the University of Oslo for financial support. The SuperSTEM Laboratory is funded by the UK Engineering and Physical Sciences Research Council (EPSRC).
*Corresponding author: danpn@fys.uio.no

${ }^{1}$ L. Pavesi and R. Turan, Silicon Nanocrystals (Wiley-VCH, Berlin, 2010), p. 1.

${ }^{2}$ G. Conibeer, Silicon Nanocrystals (Wiley-VCH, Berlin, 2010), p. 555.

${ }^{3}$ Y. Kayanuma, Phys. Rev. B 38, 9797 (1988).

${ }^{4}$ C. Bulutay and S. Ossicini, Silicon Nanocrystals (Wiley-VCH, Berlin, 2010), p. 5.

${ }^{5}$ A. D. Yoffe, Adv. Phys. 42, 173 (1993).

${ }^{6}$ E. G. Barbagiovanni, D. J. Lockwood, P. J. Simpson, and L. V. Goncharova, J. Appl. Phys. 111, 034307 (2012).

${ }^{7}$ R. Erni and N. D. Browning, Ultramicroscopy 107, 267 (2007).

${ }^{8}$ T. Hanrath and B. A. Korgel, Nano Lett. 4, 1455 (2004).

${ }^{9}$ P. D. Nguyen, D. M. Kepaptsoglou, Q. M. Ramasse, and A. Olsen, Phys. Rev. B 85, 085315 (2012).

${ }^{10}$ C. Bostedt, T. v. Buuren, T. M. Willey, N. Franco, L. J. Terminello, C. Heske, and T. Moller, Appl. Phys. Lett. 84, 4056 (2004).

${ }^{11}$ P. Tognini, L. C. Andreani, M. Geddo, A. Stella, P. Cheyssac, R. Kofman, and A. Migliori, Phys. Rev. B 53, 6992 (1996).

${ }^{12}$ J. R. Heath, J. J. Shiang, and A. P. Alivisatos, J. Chem. Phys. 101, 1607 (1994).

${ }^{13}$ D. V. Melnikov and J. R. Chelikowsky, Solid State Commun. 127, 361 (2003).

${ }^{14}$ S. Schamm, C. Bonafos, H. Coffin, N. Cherkashin, M. Carrada, G. Ben Assayag, A. Claverie, M. Tencé, and C. Colliex, Ultramicroscopy 108, 346 (2008).

${ }^{15}$ R. F. Egerton, Electron Energy-Loss Spectroscopy in the Electron Microscope (Springer, New York, 2011).

${ }^{16}$ R. Erni, N. D. Browning, Z. R. Dai, and J. P. Bradley, Micron 36, 369 (2005).

${ }^{17}$ O. L. Krivanek, G. J. Corbin, N. Dellby, B. F. Elston, R. J. Keyse, M. F. Murfitt, C. S. Own, Z. S. Szilagyi, and J. W. Woodruff, Ultramicroscopy 108, 179 (2008).

${ }^{18}$ K. Sader, B. Schaffer, G. Vaughan, R. Brydson, A. Brown, and A. Bleloch, Ultramicroscopy 110, 998 (2010).

${ }^{19}$ L. L. Araujo, R. Giulian, D. J. Sprouster, C. S. Schnohr, D. J. Llewellyn, P. Kluth, D. J. Cookson, G. J. Foran, and M. C. Ridgway, Phys. Rev. B 78, 094112 (2008).

${ }^{20}$ J. K. Bording and J. Taftø, Phys. Rev. B 62, 8098 (2000).

${ }^{21}$ N. Luca Dal, H. Sebastien, Z. Natalia, Y. Jae Hyung, W. Andrew, S. Michael, M. Jurgen, G. Giulia, and C. K. Lionel, IEEE J. Sel. Top. Quantum Electron. 12, 1151 (2006).

${ }^{22}$ S. Cosentino, S. Mirabella, M. Miritello, G. Nicotra, R. Lo Savio, F. Simone, C. Spinella, and A. Terrasi, Nanoscale Res. Lett. 6, 135 (2011).

${ }^{23}$ P. D. Nguyen, D. M. Kepaptsoglou, Q. M. Ramasse, M. F. Sunding, L. O. Vestland, T. G. Finstad, and A. Olsen, J. Appl. Phys. 112, 073514 (2012).
${ }^{24}$ A. Bianconi and R. S. Bauer, Surf. Sci. 99, 76 (1980).

${ }^{25}$ G. R. Harp, Z.-L. Han, and B. P. Tonner, Phys. Scr. 1990, 23 (1990).

${ }^{26}$ G. E. v. Dorssen, M. D. Roper, H. A. Padmore, A. D. Smith, and G. N. Greaves, Rev. Sci. Instrum. 66, 1480 (1995).

${ }^{27}$ R. Pantel, M. C. Cheynet, and F. D. Tichelaar, Micron 37, 657 (2006).

${ }^{28}$ E. D. Palik, Handbook of Optical Constants of Solids (Academic Press, MA, USA, 1991).

${ }^{29}$ J. P. R. Bolton and M. Chen, J. Phys.: Condens. Matter 7, 3373 (1995).

${ }^{30}$ J. P. R. Bolton and M. Chen, Ultramicroscopy 60, 247 (1995).

${ }^{31}$ R. Erni and N. D. Browning, Ultramicroscopy 108, 84 (2008).

${ }^{32}$ R. Erni, S. Lazar, and N. D. Browning, Ultramicroscopy 108, 270 (2008).

${ }^{33}$ C. W. Teng, J. F. Muth, R. M. Kolbas, K. M. Hassan, A. K. Sharma, A. Kvit, and J. Narayan, Appl. Phys. Lett. 76, 43 (2000).

${ }^{34}$ S. Hayashi, M. Fujii, and K. Yamamoto, Jpn. J. Appl. Phys. 28, L1464 (1989).

${ }^{35}$ E. J. Friebele, D. L. Griscom, M. Stapelbroek, and R. A. Weeks, Phys. Rev. Lett. 42, 1346 (1979).

${ }^{36}$ D. L. Griscom and E. J. Friebele, Phys. Rev. B 34, 7524 (1986).

${ }^{37}$ A. Kameyama, A. Yokotani, and K. Kurosawa, J. Appl. Phys. 95, 4000 (2004).

${ }^{38}$ R. Guerra, M. Marsili, O. Pulci, and S. Ossicini, Phys. Rev. B 84, 075342 (2011).

${ }^{39}$ C. E. Bottani, C. Mantini, P. Milani, M. Manfredini, A. Stella, P. Tognini, P. Cheyssac, and R. Kofman, Appl. Phys. Lett. 69, 2409 (1996).

${ }^{40}$ H. C. Weissker, J. Furthmüller, and F. Bechstedt, Phys. Rev. B 65, 155328 (2002).

${ }^{41}$ C. Bulutay, Phys. Rev. B 76, 205321 (2007).

${ }^{42}$ M. Mitome, Y. Yamazaki, H. Takagi, and T. Nakagiri, J. Appl. Phys. 72, 812 (1992).

${ }^{43}$ S. Takeoka, M. Fujii, S. Hayashi, and K. Yamamoto, Phys. Rev. B 58, 7921 (1998).

${ }^{44}$ Y. M. Niquet, G. Allan, C. Delerue, and M. Lannoo, Appl. Phys. Lett. 77, 1182 (2000).

${ }^{45}$ J. E. Chang et al., J. Phys. D: Appl. Phys. 45, 105303 (2012).

${ }^{46}$ D. König, J. Rudd, M. A. Green, and G. Conibeer, Phys. Rev. B 78, 035339 (2008)

${ }^{47}$ M. V. Wolkin, J. Jorne, P. M. Fauchet, G. Allan, and C. Delerue, Phys. Rev. Lett. 82, 197 (1999).

${ }^{48}$ Z. Zhou, L. Brus, and R. Friesner, Nano Lett. 3, 163 (2003).

${ }^{49}$ Y. Maeda, Phys. Rev. B 51, 1658 (1995).

${ }^{50}$ L. A. J. Garvie, P. Rez, J. R. Alvarez, P. R. Buseck, A. J. Craven, and R. Brydson, Am. Mineral. 85, 732 (2000). 